\title{
Update on Pediatric Atopic Dermatitis
}

\author{
Alexis Tracy, MD; Safiyyah Bhatti, MD; Lawrence F. Eichenfield, MD
}

\section{PRACTICE POINTS}

- There has been tremendous growth in our understanding of atopic dermatitis, with further insight into epidemiology, the impact on quality of life of affected individuals and their families, best bathing practices, and expanding treatment options.

- There are several novel topical and systemic agents recently approved and in late-stage clinical development programs that are evolving therapeutic approaches to pediatric disease.
Recent studies have led to new insights into atopic dermatitis (AD) pathogenesis and epidemiology as well as its impact on the quality of life of affected children and adolescents. In addition, there are several novel topical and systemic agents recently approved and in late-stage clinical development programs. Epidemiologic insights include relative prevalence rates in different countries and studies of subsets of pediatric patients with different disease longevity and persistence. Studies on quality of life have shown tremendous impact on sleep, not only in affected individuals but in their parents/guardians. The impact of bathing regimens is discussed. Newer topical therapies are reviewed, including topical crisaborole, with a new indication for infants as young as 3 months of age. Topical Janus kinase (JAK) inhibitors are being developed, with some studies including adolescents and children. Other novel therapies include the topical aryl hydrocarbon receptor agonist tapinarof and oral JAK inhibitors; adolescents are being included in the initial clinical trials for several of these therapies. Dupilumab, the first biologic agent approved for $A D$, has now been well studied in patients aged 6 years and older, with expanded indication down to 6 years of age.

Cutis. 2020;106:143-146. $\bigwedge$ topic dermatitis (AD) is a chronic, pruritic, inflammatory skin disease that occurs most frequently in children but also affects many adolescents and adults. There has been a tremendous evolution of knowledge in $\mathrm{AD}$, with insights into pathogenesis, epidemiology, impact of disease, and new therapies. A variety of studies examine the epidemiology of $\mathrm{AD}$ and associated comorbidities. The broad developments in disease state research are reflected in new publication numbers of AD citations on PubMed. A PubMed search of articles indexed for MEDLINE at the end of 2010 using the term atopic dermatitis would have shown 965 citations during the preceding 1-year period. In the 1-year period of June 2019 to June 2020, there were more than 2000 articles. The large body of research includes work of great significance in pediatric $\mathrm{AD}$, and in this article we review recent findings that are important in understanding the progress being made in the field.

\section{Epidemiology and Comorbidities}

The epidemiology of AD has evolved over the last few decades, with emerging trends and novel insights into the burden of disease. ${ }^{1}$ In a recent cross-sectional study on the epidemiology of AD in children aged 6 to 11 years, the 1-year diagnosed $\mathrm{AD}$ prevalence estimates worldwide included the following: United States, $10.0 \%$; Canada, 13.3\%; the EU5 Countries, 15.5\%; Japan, 10.3\%; and all countries studied, $12.2 \% .^{2}$ Another recent paper that analyzed data from the Fragile Families and Child Wellbeing Study showed that the prevalence and persistence of $\mathrm{AD}$ in urban US children was $15.0 \% .^{3}$ Although pediatric AD

From the Division of Pediatric Dermatology, Departments of Dermatology and Pediatrics, University of California, San Diego, and Rady Children's Hospital, San Diego. Drs. Tracy and Bhatti also are from the Division of Allergy and Immunology.

Drs. Tracy and Bhatti have been investigators for the following companies on behalf of their institution but received no compensation: Abbvie; Incyte Corporation; Regeneron Pharmaceuticals, Inc; and Valeant Pharmaceuticals International Inc. Dr. Eichenfield is an investigator for AbbVie; LEO Pharma; Pfizer Inc; Regeneron Pharmaceuticals, Inc; and Sanofi Genzyme. He also is a consultant for Almirall; Dermavant Sciences Ltd; Dermira, Inc; DS Biopharma; Eli Lilly and Company; Forte Biopharma; Galderma Laboratories, LP; Incyte Corporation; LEO Pharma; Novartis; Ortho Dermatologics; Otsuka Pharmaceutical; Pfizer Inc; Regeneron Pharmaceuticals, Inc; and Sanofi Genzyme.

Correspondence: Lawrence F. Eichenfield, MD, 3020 Children's Way, Mail Code 5092, San Diego, CA 92123 (Leichenfield@rchsd.org).

doi:10.12788/cutis.0077 
may spontaneously remit over time, disease continuing into adolescence and adulthood is common. Paternoster et $\mathrm{al}^{4}$ studied the longitudinal course of $\mathrm{AD}$ in children from 2 birth cohort prospective studies, showing distinct $\mathrm{AD}$ phenotypes having differing course trajectories over time. Disease subsets included patients with earlyonset-persistent and early-onset-late-resolving disease. ${ }^{4}$ Whether phenotyping or subgroup analysis can be used to predict disease course or risk for development of comorbidities is unknown, but it is interesting to consider how such work could influence tailoring of specific therapies to early disease presentation.

Atopic dermatitis poses a serious public health burden owing to its high prevalence, considerable morbidity and disability, increased health care utilization, and cost of care. ${ }^{1}$ Recent studies have found notably higher rates of multiple medical and mental health comorbidities in both children and adults with $\mathrm{AD}$, including infections, atopic comorbidities (eg, allergic rhinitis, asthma, food allergies), eye diseases (eg, keratitis, conjunctivitis, keratoconus), and possible cardiovascular diseases and autoimmune disorders. ${ }^{1,5-9}$ Allergic comorbidities are quite common in pediatric $\mathrm{AD}$ patients. ${ }^{10} \mathrm{In}$ a recent study examining the efficacy and safety of dupilumab monotherapy in 251 adolescents with moderate to severe inadequately controlled $\mathrm{AD}$, most had comorbid type 2 diseases including asthma (53.6\%), food allergies $(60.8 \%)$, and allergic rhinitis $(65.6 \%) .{ }^{11}$

\section{Quality of Life/Life Impact of AD}

Pediatric AD has a major impact on the quality of life of patients and their families. ${ }^{12}$ The well-being and development of children are strongly influenced by the physical and psychosocial health of parents/guardians. Two studies by Ramirez and colleagues ${ }^{13,14}$ published in 2019 examined sleep disturbances and exhaustion in mothers of children with AD. Data for the studies came from the Avon Longitudinal Study of Parents and Children. Children with active $\mathrm{AD}$ reported worse sleep quality than those without AD, with nearly $50 \%$ higher odds of sleep-quality disturbances. Analysis of the cohort data from 11,649 mother-child pairs who were followed up with a time-varying measure of child $\mathrm{AD}$ activity and severity as well as self-reported maternal sleep measures repeated at multiple time points for children aged 6 months to 11 years showed that mothers of children with $\mathrm{AD}$ reported difficulty falling asleep, subjectively insufficient sleep, and daytime exhaustion throughout the first 11 years of childhood. ${ }^{13,14}$ These data suggest that sleep disturbance may be a family affair.

A cross-sectional, real-world study on the burden of $\mathrm{AD}$ in children aged 6 to 11 years assessed by selfreport demonstrated a substantial and multidimensional impact of $\mathrm{AD}$, including itch, sleep disturbance, skin pain, and health-related quality-of-life impact, as well as comorbidities and school productivity losses. The burden associated with $\mathrm{AD}$ was remarkable and increased with disease severity. ${ }^{15}$
Drucker et $\mathrm{al}^{16}$ completed a comprehensive literature review on the burden of $\mathrm{AD}$, summarized as a report for the National Eczema Association. Quality-of-life impact on pediatric patients included high rates of emotional distress; social isolation; depression; limitations in activities due to lesions with fear of triggers; and behavioral problems such as irritability, crying, and sleep disturbance resulting in difficulty performing at school. ${ }^{16}$ The psychological impact on children as well as emotional and behavioral difficulties may impact the ability for parents/ guardians to implement treatment plans. ${ }^{17}$

There is a striking association between mental health disorders and $\mathrm{AD}$ in the US pediatric population, with a clear dose-dependent relationship that has been observed between the prevalence of a mental health disorder and the reported severity of the skin disease. Data suggest children with AD may be at increased risk for developing mental health disorders. The National Survey of Children's Health found statistically significant increases in the likelihood of attention deficit hyperactivity disorder (odds ratio [OR], 1.87), depression (OR, 1.81), anxiety (OR, 1.77), conduct disorder (OR, 1.87), and autism $(\mathrm{OR}, 3.04){ }^{6}$

\section{Evolving Practices and Therapies}

Bathing Practices-There has long been much controversy regarding best bathing habits for patients with AD. In a 2009 study, cutaneous hydration was quantified after various bathing and moisturizing regimens. ${ }^{18}$ The study showed clear benefits of emollient application on skin hydration, either after bathing or without bathing. Bathing followed by emollient applications did not decrease skin hydration in contrast to bathing without emollient application. ${ }^{18}$

There are limited studies evaluating bathing frequency in pediatric patients, and many families receive conflicting information regarding best practice. In one study that surveyed 354 parents, more than $75 \%$ of parents/guardians who had seen multiple providers for their child's AD reported a substantial amount of confusion and frustration from conflicting advice on bathing frequency. ${ }^{19}$ Cardona et $\mathrm{al}^{20}$ undertook a randomized clinical trial of frequent bathing and moisturizing vs less-frequent bathing and moisturizing in pediatric patients with $\mathrm{AD}$ aged 6 months to 11 years. Patients were divided into 2 groups: 1 being bathed twice daily with immediate moisturizer application and the other being bathed twice weekly followed by moisturization, then a switch to the other method. Patients used standardized topical corticosteroids (TCSs) in both groups. There were significant improvements in scoring $\mathrm{AD}$ and other objective measures during the frequent bathing time period vs infrequent bathing; in the group that bathed more frequently, SCORAD (SCORing Atopic Dermatitis) decreased by 21.2 compared with the group that bathed less frequently ( $95 \%$ confidence interval, 14.9-27.6; $P<.0001)$. These findings suggest that more-frequent bathing with immediate moisturization is 
superior as an acute treatment intervention for improving $\mathrm{AD}$ disease severity in comparison to less-frequent bathing with immediate moisturization. ${ }^{20}$

\section{Expanding Treatment Options}

Topical Phosphodiesterase Inhibitors-There are several new and evolving topical therapies in AD. Crisaborole ointment $2 \%$ is a steroid-free phosphodiesterase inhibitor approved in 2016 by the US Food and Drug Administration (FDA) for mild to moderate $\mathrm{AD}$ in patients aged 2 years and older. A recent multicenter, open-label, single-arm study in 137 infants (CrisADe CARE 1) evaluated the pharmacokinetics and efficacy of crisaborole ointment $2 \%$ applied twice daily for 4 weeks in pediatric patients aged 3 months to less than 24 months of age with mild to moderate AD. ${ }^{21}$ The study had 2 cohorts: one with a minimum of $5 \%$ body surface area involvement and another (the pharmacokinetic cohort) with a minimum of $35 \%$ body surface area involvement. Both cohorts demonstrated similar efficacy data. From baseline to day 29, the mean percentage change in eczema area and severity index (EASI) score was $-57.5 \%$, and an investigator global assessment (IGA) score of clear or almost clear with at least a 2-grade improvement was achieved in $30.2 \%$ of patients. Crisaborole systemic exposures in infants were comparable with those in patients aged 2 years or older. Patients tolerated crisaborole well, with a $4 \%$ rate of burning, which was similar to other studies in children and adults but perhaps lower than seen in clinical practice. Pharmacokinetic studies did not show any remarkable noticeable concern with accumulation of propylene glycol absorption. ${ }^{21}$

Based on the CrisADe CARE 1 study data, in March 2020 the FDA extended the indication of crisaborole ointment $2 \%$ from a prior lower age limit of 24 months to approval for use in treating mild to moderate AD in children as young as 3 months, making it the first nonsteroidal topical anti-inflammatory medication to be approved in children younger than 2 years in the United States.

\section{Evolving Topical Therapies}

Topical Janus Kinase Inhibitors-Ruxolitinib is a potent inhibitor of Janus kinase 1 (JAK-1) and Janus kinase 2 (JAK-2) and has been developed in topical formulations. In recent phase 3 clinical trials of patients with $\mathrm{AD}$ aged 12 years and older with mild to moderate disease (TRuE-AD1 and TRuE-AD2), more than half of the patients treated with either ruxolitinib cream in a $0.75 \%$ or $1.5 \%$ concentration reached EASI- 75 after 8 weeks of treatment. ${ }^{22}$ Additionally, more patients treated with topical ruxolitinib reached an IGA score of clear to almost clear than patients treated with vehicle at the end of treatment. Thus far, it appears to be very well tolerated, significantly decreases EASI score $(P<.0001)$, and improves overall pruritus. ${ }^{22}$

Delgocitinib is a topical pan-JAK inhibitor that blocks several cytokine-signaling cascade pathways. It was first developed and approved in Japan in an ointment formulation for use in patients with $\mathrm{AD}$ aged 16 years and older. ${ }^{23}$ The efficacy and safety profile of delgocitinib is currently being evaluated in pediatric patients with $\mathrm{AD}$ in Japan. In a recent phase 2 clinical study of 103 Japanese patients aged 2 to 15 years with moderate to severe $\mathrm{AD}$, patients were randomized to receive either delgocitinib ointment in $0.25 \%$ or $0.5 \%$ concentrations or vehicle ointment twice daily for 4 weeks. The proportion of patients with a modified EASI-75 score was $38.2 \%(13 / 34)$ in the $0.25 \%$ group and $50.0 \%$ $(17 / 34)$ in the $0.5 \%$ group vs $8.6 \%(3 / 35)$ in the placebo group. More patients treated with delgocitinib ointment received an IGA score of clear or almost clear than patients treated with vehicle at the end of treatment. Overall, both delgocitinib groups demonstrated superior improvement in clinical symptoms and signs without notable side effects. ${ }^{24}$

Tapinarof-Tapinarof is a topical therapeutic aryl hydrocarbon receptor agonist. In a recent phase 2 randomized study of 2 concentrations and 2 frequencies of tapinarof cream vs vehicle in 247 randomized patients aged 12 to 65 years with moderate to severe disease, tapinarof demonstrated greater success with both concentrations than vehicle at all visits beyond week $2 .{ }^{25}$ Additionally, in patients treated with tapinarof cream 1\%, nearly $50 \%$ reached an IGA score of clear to almost clear with at least a 2-grade improvement. More than 50\% of patients achieved EASI-75 improvement at 12 weeks of treatment with tapinarof cream 1\% used daily. These findings suggest that tapinarof may be an efficacious and well-tolerated treatment for both adolescents and adults with $\mathrm{AD}$; however, large confirmation trials are needed to further investigate. ${ }^{25}$

\section{Systemic Treatments}

Oral JAK Inhibitors-Some of the most exciting novel therapies include several oral JAK inhibitors that target different combinations of kinases and have been shown to decrease $\mathrm{AD}$ severity and symptoms. Some of these agents have indications in other disease states, such as baricitinib and upadacitinib, which are both FDA approved for the treatment of rheumatoid arthritis, whereas others, such as abrocitinib, have been studied specifically for $\mathrm{AD}$.

Although some agents have only been studied in adults to date, others have included adolescents in their core studies, such as abrocitinib, which received Breakthrough Therapy designation from the FDA for the treatment of patients with moderate to severe $\mathrm{AD}$ in February 2018. In recent phase 3 trials of patients aged 12 years and older with moderate to severe AD (JADE MONO-1 and JADE MONO-2), both doses of abrocitinib improved the IGA and EASI-75 outcomes compared with placebo. ${ }^{26}$ Additional studies will be conducted to further investigate the relative efficacy and safety in patients younger than 18 years. 
Biologics-Dupilumab is a fully human monoclonal antibody that inhibits IL-4 and IL-13 signaling without suppressing the immune system. It is approved for use in patients aged 12 years and older with moderate to severe asthma and in adults with chronic rhinosinusitis with nasal polyposis. It is the first biologic to show positive results in the moderate to severe pediatric $\mathrm{AD}$ population. There are now extended data available exhibiting sustained benefit in adolescent patients who were continued on dupilumab therapy, evidenced by further improvement in EASI scores at the 1-year mark. ${ }^{27}$

Recently, dupilumab received approval for use in patients aged 6 to 11 years, making it the first biologic for $\mathrm{AD}$ to be approved for use in patients younger than 12 years. The expedited FDA approval was based on the phase 3 results in which the efficacy and safety of dupilumab combined with TCSs were compared to TCSs alone $(\mathrm{N}=367) .{ }^{28}$ In this trial, more than twice as many children achieved clear or almost clear skin and more than 4 times as many achieved itch reduction with dupilumab plus TCSs than with TCSs alone. Three-quarters of patients receiving dupilumab at the subsequently approved dosing achieved at least a $75 \%$ improvement in overall disease. ${ }^{28}$ An additional study is being conducted that includes pediatric patients aged 6 months to younger than 6 years (ClinicalTrials.gov Identifier NCT03346434).

\section{Future Directions in Pediatric AD}

Our review summarizes only some of the agents under clinical investigation for use in pediatric AD. Early treatment to establish excellent long-term disease control with aggressive topical regimens or with systemic agents may alter the course of $\mathrm{AD}$ and influence the development of comorbidities, though this has not yet been shown in clinical studies. The long-term impact of early treatment, along with many other intriguing issues, will be studied more in the near future.

\section{REFERENCES}

1. Silverberg JI. Public health burden and epidemiology of atopic dermatitis. Dermatol Clin. 2017;35:283-289.

2. Silverberg JI, Barbarot S, Gadkari A, et al. Epidemiology of atopic dermatitis in children aged 6-11 years: a cross-sectional study in the United States (US), Canada, Europe, and Japan. Paper presented at: American Academy of Dermatology Annual Meeting; March 20-24, 2020; Denver, CO.

3. McKenzie C, Silverberg JI. The prevalence and persistence of atopic dermatitis in urban United States children. Ann Allergy Asthma Immunol. 2019;123:173-178.e1.

4. Paternoster L, Savenije OEM, Heron J, et al. Identification of atopic dermatitis subgroups in children from 2 longitudinal birth cohorts. J Allergy Clin Immunol. 2018;141:964-971.

5. Silverberg JI, Simpson EL. Association between severe eczema in children and multiple comorbid conditions and increased healthcare utilization. Pediatr Allergy Immunol. 2013;24:476-486.

6. Yaghmaie P, Koudelka CW, Simpson EL. Mental health comorbidity in patients with atopic dermatitis. J Allergy Clin Immunol. 2013;131:428-433.

7. Narla S, Silverberg JI. Association between childhood atopic dermatitis and cutaneous, extracutaneous and systemic infections. $\mathrm{Br}$ J Dermatol. 2018;178:1467-1468.
8. Thyssen JP, Toft PB, Halling-Overgaard AS, et al. Incidence, prevalence, and risk of selected ocular disease in adults with atopic dermatitis. J Am Acad Dermatol. 2017;77:280-286.

9. Standl M, Tesch F, Baurecht H, et al. Association of atopic dermatitis with cardiovascular risk factors and diseases. J Invest Dermatol. 2017;137:1074-1081.

10. Paller A, Jaworski JC, Simpson EL, et al. Major comorbidities of atopic dermatitis: beyond allergic disorders. Am J Clin Dermatol. 2018;19:821-838.

11. Simpson EL, Paller AS, Siegfried EC, et al. Efficacy and safety of dupilumab in adolescents with uncontrolled moderate to severe atopic dermatitis. JAMA Dermatol. 2019;156:44-56.

12. Pustišek N, Vurnek Živkovs M, Šitum M. Quality of life in families with children with atopic dermatitis. Pediatr Dermatol. 2016;33:28-32.

13. Ramirez FD, Chen S, Langan SM, et al. Assessment of sleep disturbances and exhaustion in mothers of children with atopic dermatitis. JAMA Dermatol. 2019;155:556-563.

14. Ramirez FD, Chen S, Langan SM, et al. Association of atopic dermatitis with sleep quality in children. JAMA Pediatr. 2019;173:e190025.

15. Weidinger S, Simpson EL, Eckert L, et al. The patient-reported disease burden in pediatric patients with atopic dermatitis: a cross-sectional study in the United States (US), Canada, Europe, and Japan. Paper presented at: American Academy of Dermatology Annual Meeting; March 20-24, 2020; Denver, CO.

16. Drucker AM, Wang AR, Li WQ, et al. The burden of atopic dermatitis: summary of a report for the National Eczema Association. I Invest Dermatol. 2017;137:26-30.

17. Mitchell AE. Bidirectional relationships between psychological health and dermatological conditions in children. Psychol Res Behav Manag. 2018;11:289-298.

18. Chiang C, Eichenfield LF. Quantitative assessment of combination bathing and moisturizing regimens on skin hydration in atopic dermatitis. Pediatr Dermatol. 2009;26:273-278.

19. Kempe E, Jain N, Cardona I. Bathing frequency recommendations for pediatric atopic dermatitis: are we adding to parental frustration? Ann Allergy Asthma Immunol. 2013;111:298-299.

20. Cardona ID, Kempe EE, Lary C, et al. Frequent versus infrequent bathing in pediatric atopic dermatitis: a randomized clinical trial. J Allergy Clin Immunol Pract. 2020;8:1014-1021.

21. Schlessinger J, Shepard JS, Gower R, et al. Safety, effectiveness, and pharmacokinetics of crisaborole in infants aged 3 to $<24$ months with mild-to-moderate atopic dermatitis: a phase IV open-label study (CrisADe CARE 1). Am J Clin Dermatol. 2020;21:275-284.

22. Papp K, Szepietowski JC, Kircik L, et al. Efficacy and safety of ruxolitinib cream for the treatment atopic dermatitis: results from two phase 3, randomized, double-blind studies. Presented at: 2nd Annual Revolutionizing Atopic Dermatitis Conference; April 5, 2020; Chicago, IL.

23. Dhillon S. Delgocitinib: first approval. Drugs. 2020;80:609-615.

24. Nakagawa H, Nemoto O, Igarashi A, et al. Phase 2 clinical study of delgocitinib ointment in pediatric patients with atopic dermatitis. J Allergy Clin Immunol. 2019;144:1575-1583.

25. Peppers J, Paller AS, Maeda-Chubachi T, et al. A phase 2, randomized dose-finding study of tapinarof (GSK2894512 cream) for the treatment of atopic dermatitis. J Am Acad Dermatol. 2019;80:89-98.e3.

26. Simpson EL, Sinclair R, Forman S, et al. Efficacy and safety of abrocitinib in adults and adolescents with moderate-to-severe atopic dermatitis (JADE MONO-1): a multicentre, double-blind, randomised, placebo-controlled, phase 3 trial. Lancet. 2020;396:255-266.

27. Cork MJ, Thaçi D, Eichenfield LF, et al. Dupilumab in adolescents with uncontrolled moderate-to-severe atopic dermatitis: results from a phase IIa open-label trial and subsequent phase III open-label extension. Br J Dermatol. 2020;182:85-96.

28. Paller AS, Siegfried EC, Thaçi D, et al. Efficacy and safety of dupilumab with concomitant topical corticosteroids in children 6 to 11 years old with severe atopic dermatitis: a randomized, double-blinded, placebocontrolled phase 3 trial [published online June 20, 2020]. J Am Acad Dermatol. doi:10.1016/j.jaad.2020.06.054. 